\title{
Prophylaxe
}

\section{NEU: Mundwasser für eine optimierte orale Antisepsis}

Für eine optimierte orale Antisepsis sowohl in der Zahnarztpraxis als auch für die Anwendung durch den Patienten zuhause bietet Sunstar Deutschland ab sofort das freiverkäufliche Arzneimittel PAROEX 1,2mg/ $\mathrm{ml}$ Mundwasser an. Es enthält als Wirkstoff $0,12 \%$ Chlorhexidin (CHX) und ist angezeigt bei Infektionen der Mundschleimhaut sowie zur postoperativen Versorgung nach dentalchirurgischen Eingriffen bei Er- wachsenen und Kindern über 6 Jahren. Das Mundwasser ist sowohl in der wirtschaftlichen 51-Maxi-Flasche mit hygienischem Pumpspender für die Anwendung in der Zahnarztpraxis verfügbar, als auch in der 300 ml-Flasche mit praktischem Dosierbecher für die Fortsetzung der Behandlung durch den Patienten zuhause. Der besonders angenehme Geschmack unterstützt die Compliance und fördert damit den Therapieerfolg.

Nach einer Pressemitteilung der Sunstar Deutschland GmbH, Schönau 